\title{
Is the Therapeutic Drug Monitoring of Anti-TNF Agents Necessary in Korean Inflammatory Bowel Disease Patients?
}

\author{
Chang Soo Eun \\ Department of Internal Medicine, Hanyang University Guri Hospital, Guri, Korea
}

See "Clinical Use of Measuring Trough Levels and Antibodies against Infliximab in Patients with Pediatric Inflammatory Bowel Disease" by So Yoon Choi, et al. on page 55, Vol. 11. No. 1, 2017

Inflammatory bowel diseases (IBD), including Crohn's disease and ulcerative colitis, are idiopathic chronic intestinal inflammatory diseases, characterized by recurrent episodes of relapse and remission of disease. The introduction of anti-tumor necrosis factor (anti-TNF) agents has greatly improved the treatment outcomes for IBDs, enabling higher rates of clinical and endoscopic remission in patients who are refractory to conventional therapies such as corticosteroids and thiopurines. ${ }^{1,2}$ Despite the proven therapeutic efficacy of anti-TNF agents, treatment failures to those sometimes occur in the form of primary nonresponse or secondary loss of response (LOR).

About $10 \%$ to $20 \%$ of IBD patients have been reported to be unresponsive to induction treatment with anti-TNF agents. ${ }^{3}$ The mechanism of this primary nonresponse is unclear. Meanwhile, of the patients who initially respond to anti-TNF treatment, a significant proportion loses response over time with annual risk of $13 \%$ to $24 \%{ }^{4}$ Several distinct mechanisms for secondary LOR have been suggested, including the formation of antidrug antibodies (ADA), nonimmune drug clearance, relentless TNFmediated flare consuming the anti-TNF agent, shift of disease pathway away from TNF, as well as non-IBD related mechanisms such as irritable bowel syndrome, infection, cancer, and so forth. ${ }^{5}$ To prevent LOR to anti-TNF treatment, scheduled treatment rather than episodic therapy and combination therapy with immunomodulators than anti-TNF monotherapy are recommended. $^{6}$

In patients presenting with LOR to anti-TNF agents, after excluding non-IBD, noninflammatory causes, several therapeutic options such as dose intensification, switching to another antiTNF agents, and switching to another biologics with different mechanism of action would be considered. In these circumstances, pharmacokinetic measurements of anti-TNF trough levels (TL) and ADA could be useful to elucidate mechanisms of LOR and guide selection of most appropriate therapeutic strategy. ${ }^{3}$

Several studies have demonstrated clinical importance of therapeutic drug monitoring (TDM) of anti-TNF agents by determining drug levels and ADA in terms of a tailored approach to IBD patients. Higher anti-TNF TLs have been associated with better clinical outcomes and endoscopic improvement, while undetectable anti-TNF TLs were related to loss of clinical response and higher risk for surgery. On the contrary, immunogenicity (formation of ADA) has been known to accelerate antiTNF clearance and increase the risk of infusion reaction to antiTNF agents, although the exact clinical impact of ADA on antiTNF efficacy is still controversial. ${ }^{7}$ In Western countries, clinical decision algorithms with TDM based on drug levels and ADA for the management of IBD patients with LOR, to optimize the efficacy of anti-TNF and then to reduce health-related cost, has been proposed and widely performed in clinical practice. ${ }^{8}$ In addition, some researchers have suggested that proactive TDM will be promising even for IBD patients in clinical remission, to maintain optimal anti-TNF concentrations and then to reduce costs and risk for adverse events. ${ }^{7}$

However, most of these studies have been performed in Western countries. To date, there has been no data regarding TDM and its impact on clinical outcomes and medical costs in Korean IBD patients with secondary LOR. And, measurements of drug levels and ADA are not yet available in Korea.

In this issue of Gut and Liver, Choi et al. ${ }^{9}$ investigated the

Correspondence to: Chang Soo Eun

Department of Internal Medicine, Hanyang University Guri Hospital, 153 Gyeongchun-ro, Guri 11923, Korea

Tel: +82-31-560-2228, Fax: +82-31-555-2998, E-mail: cseun@hanyang.ac.kr

pISSN 1976-2283 eISSN 2005-1212 https://doi.org/10.5009/gnl16574

(a) This is an Open Access article distributed under the terms of the Creative Commons Attribution Non-Commercial License (http://creativecommons.org/licenses/by-nc/4.0) which permits unrestricted non-commercial use, distribution, and reproduction in any medium, provided the original work is properly cited. 
clinical usefulness of infliximab (IFX) TLs and ADA in 39 pediatric IBD patients in a retrospective manner and proposed a tailored therapeutic strategy for IFX therapy based on IFX TLs and ADA status in patients with LOR. They compared serum IFX TLs and ADA between 16 patients with clinical remission and 23 patients with a poor response to IFX treatment and remeasured the IFX TLs and ADA after IFX dose intensification in 21 patients with LOR. The authors showed that the IFX TLs were significantly higher in patients with clinical remission than those with a poor response (median, $3.99 \mu \mathrm{g} / \mathrm{mL}$ vs $0.88 \mu \mathrm{g} / \mathrm{mL}$; $\mathrm{p}=0.002$ ) and the ADA was detectable in only patients with LOR $(7 / 23,30.4 \%)$. In addition, after IFX dose intensification in patients with LOR, the IFX TLs were increased in 17 patients with improved response, while four patients with no response have no detectable IFX but detectable ADA. This study, firstly evaluated the efficacy of anti-TNF TLs and ADA in Korean IBD patients, provide a valuable clinical insight into the TDM of antiTNF agents for IBD patients with secondary LOR in Korea.

However, as the authors described, this study performed retrospectively with a small sample size and did not evaluate clinical outcomes and response to dose intensification of IFX in the long-term. To validate the results of this study, especially in adult patients, a prospective multicenter long-term clinical trial with a larger sample size in Korean IBD patients with anti-TNF treatments should be conducted. One of these would undoubtedly be the OACIS study from KASID (Korean association for the study of intestinal diseases), a prospective, openlabel, multicenter trial for the occurrence of ADA and change of drug level after anti-TNF therapy and their impact on clinical outcomes in moderate to severe IBD.

\section{CONFLICTS OF INTEREST}

No potential conflict of interest relevant to this article was reported.

\section{REFERENCES}

1. Lee HA, Suk JY, Choi SY, et al. Characteristics of pediatric inflammatory bowel disease in Korea: comparison with EUROKIDS data. Gut Liver 2015;9:756-760.

2. Kim MJ, Lee WY, Choe YH. Expression of TIM-3, human betadefensin-2, and FOXP3 and correlation with disease activity in pediatric Crohn's disease with infliximab therapy. Gut Liver 2015;9:370-380.

3. Ben-Horin S, Chowers Y. Tailoring anti-TNF therapy in IBD: drug levels and disease activity. Nat Rev Gastroenterol Hepatol 2014;11:243-255.

4. Billioud V, Sandborn WJ, Peyrin-Biroulet L. Loss of response and need for adalimumab dose intensification in Crohn's disease: a systematic review. Am J Gastroenterol 2011;106:674-684.

5. Ben-Horin S, Kopylov U, Chowers Y. Optimizing anti-TNF treatments in inflammatory bowel disease. Autoimmun Rev 2014;13: 24-30.

6. Lee KM, Kim YS, Seo GS, Kim TO, Yang SK; IBD Study Group of the Korean Association for the Study of Intestinal Diseases. Use of thiopurines in inflammatory bowel disease: a consensus statement by the Korean Association for the Study of Intestinal Diseases (KASID). Intest Res 2015;13:193-207.

7. Felice C, Marzo M, Pugliese D, et al. Therapeutic drug monitoring of anti-TNF-alpha agents in inflammatory bowel diseases. Expert Opin Biol Ther 2015;15:1107-1117.

8. Khanna R, Sattin BD, Afif W, et al. Review article: a clinician's guide for therapeutic drug monitoring of infliximab in inflammatory bowel disease. Aliment Pharmacol Ther 2013;38:447-459.

9. Choi SY, Kang B, Lee JH, Choe YH. Clinical use of measuring trough levels and antibodies against infliximab in patients with pediatric inflammatory bowel disease. Gut Liver 2017;11:55-61. 